\title{
Integrating people and place: A density-based measure for assessing accessibility to opportunities
}

\author{
* Mark W. Horner ${ }^{a}$ \\ Florida State University
}

\author{
Joni A. Downs ${ }^{b}$ \\ University of South Florida
}

\begin{abstract}
Mobile object analysis is a well-studied area of transportation and geographic information science (GIScience). Mobile objects may include people, animals, or vehicles. Time geography remains a key theoretical framework for understanding mobile objects' movement possibilities. Recent efforts have sought to develop probabilistic methods of time geography by exploring questions of data uncertainty, spatial representation, and other limitations of classical approaches. Along these lines, work has blended time geography and kernel density estimation in order to delineate the probable locations of mobile objects in both continuous and discrete network space. This suite of techniques is known as time geographic density estimation (TGDE). The present paper explores a new direction for TGDE, namely the creation of a density-based accessibility measure for assessing mobile objects' potential for interacting with opportunity locations. As accessibility measures have also garnered widespread attention in the literature, the goal here is to understand the magnitude and nature of the opportunities a mobile object had access to, given known location points and a time budget for its movement. New accessibility measures are formulated and demonstrated with synthetic trip diary data. The implications of the new measures are discussed in the context of people-based vs. placedbased accessibility analyses.
\end{abstract}

\section{Introduction}

Mobile object analysis continues to be a well-studied topic in transportation and geographic information science (GIScience) (Laube, Imfeld, and Weibel 2005; Hornsby and Egenhofer 2002; Downs and Horner 2012; Li et al. 2011; Neutens, Schwanen, and Witlox 2011). Time geography remains a key theoretical framework for understanding mobile objects' movement possibilities (Miller 2005). Within time geography, recent efforts have sought to enhance its "probabilistic" possibilities by exploring questions of data uncertainty, spatial representation, and other limitations of classical approaches (Winter and Lin 2011; Kuijpers et al. 2010; Neutens, Schwanen, and Witlox 2011). Consistent with this thrust, Downs (2010) fused time geography and kernel density estimation by developing time-geographic density estimation (TGDE), a technique that may be used to estimate mobile objects' probable locations in continuous space given a time budget between known control points (Downs 2010). Subsequent work by Downs and Horner (2012) extended TGDE to discrete network space, demonstrating its application with GPS-based vehicle tracking data. Their research developed probabilistic potential path trees, which improve upon the classical network potential path tree, which simply captures where an object such as a vehicle could have been, by differentially identifying network links as either more or less likely to have been traversed by the vehicle. Effectively, their technique delineates areas in the potential path tree where the vehicle was most likely to have traveled. Downs and Horner (2012) also discussed the nature of the opportunities (e.g., shopping, employment, etc.) within the probabilistic potential path trees computed for their study area as a way of contextualizing their results. However, there was no formal assessment made of the nature of the opportunities within the potential path trees because network-based

\footnotetext{
* Corresponding author

${ }^{a}$ mhorner@fsu.edu

bdowns@usf.edu

Copyright 2014 Mark W. Horner and Joni A. Downs.

Licensed under the Creative Commons Attribution - NonCommercial License 3.0.
} 
TGDE (per Downs and Horner 2012) has no facility for capturing this dimension of travel potential. Inclusion of such capability into TGDE would allow for the analysis of the opportunities most likely to be accessible to a vehicle (or other moving object), given its known travel itinerary.

There has been a vast amount of research conducted on accessibility and its measurement during the last several years, with many studies incorporating aspects of time geography (O'Sullivan, Morrison, and Shearer 2000; Kwan 1998; Miller 1999; Neutens et al. 2010; Delafontaine, Neutens, and Van de Weghe 2012; Kim and Kwan 2003; Levine et al. 2012; El-Geneidy and Levinson 2007; Scott and Horner 2008) Although a diversity of approaches are found in the conceptualization of accessibility (Handy and Niemeier 1997), metrics can generally be thought of as being either "place-based" or "people-based" (Miller 2007; Delafontaine, Neutens, and Van de Weghe 2012). Place-based metrics map accessibility to locations; they capture the aggregate opportunities available given a budget for travel (e.g., how many restaurants may be reached in 30 minutes by transit) while omitting individual context and personal constraints. People-based metrics account for the personal context and constraints of travelers, as metrics map to (are computed for) the individual. However, achieving aggregation of these measures, particularly aggregation that could be used to analyze trends across individuals and/or place-based policies, has remained elusive. Recent efforts have sought to bridge these two perspectives as a means of bringing both approaches to bear on accessibility (Delafontaine, Neutens, and Van de Weghe 2012), though more research is needed to address this challenge. In this paper, we will show that our TGDE-based accessibility measure blends both the person- and place-based perspectives and, as such, represents an advance in the measurement of accessibility.

The present paper explores a new direction for TGDE, namely the creation of a density-based accessibility measure for mobile objects. Our new metric gauges how accessible a moving object is to particular opportunities of interest, given the constraints inherent to its movement plan. In this way, we are able to not only visualize where the object most likely could have been (Downs and Horner 2012), but we also capture the configuration and magnitude of activities relative to the travel path. The flexible nature of the model is such that it may be used to produce aggregated summaries of accessibility for individuals as well as places, thereby bridging these two divergent perspectives.

This section has introduced the scope of our paper, and the next portion offers a brief overview of the two strands of thought guiding our work: those of time geography and accessibility modeling. Following this material, we discuss the development of our new accessibility model and demonstrate its use with a synthetic sample travel diary dataset. Lastly, we conclude with a discussion of our work and suggestions for future research.

\section{Background and concepts}

This section focuses on establishing the two major themes underpinning our work, namely time geography and accessibility. Each of these areas is briefly reviewed in turn, followed by a synthesis of selected research efforts common to both domains.

\subsection{Time geography}

The concept of time geography was introduced by Hägerstrand as a way to understand how people's movement patterns are influenced by their spatial, temporal, and personal constraints (Lenntorp 1999; Hägerstrand 1970). Spatial constraints might include the location of a person's workplace in relation to his or her home, while temporal constraints might include the time he or she must spend at each of those places. An example of a personal constraint would be the capability of the individual to travel between home and work while relying solely on public transportation. Time geography provides a way 
to explore the potential mobility of the individual given all of these limitations considered simultaneously. For example, one can identify the spatial opportunities (e.g., grocery stores, entertainment venues, health clinics) that an individual can possibly access given known home and work locations, the allowable travel time between them, and the mode of travel. If this type of exercise is repeated for a number of individuals, we can examine how people choose to move about space, as well as understand how those choices are limited by the particular constraints each individual faces.

At a basic level, the type of analysis described above can be accomplished quantitatively through the construction of key elements of time geography: the space-time path and space-time prism. These fundamental constructs are mathematically defined and described in detail by Miller (2005). The spacetime path traces the movement of a person or other mobile object through space and time by estimating the trajectory taken between known locations. These locations may be represented either as specific recorded activities, such as work or shopping, or arbitrarily reported locations such as those collected by GPS receivers. In Euclidean space, the space-time path is generated by connecting consecutive locations with straight lines; in network space, it is modeled by connecting them with shortest paths along the network (Downs 2010). The space-time path anchors the space-time prism, which maps the potential movements of an object in both space and time given information about its movement constraints. For any point in time, these possible locations comprise what is termed the potential path area in Euclidean space or a potential path tree in network space. Collectively, space-time paths and prisms have been used to study a wide range of topics related to transportation, such as analyzing human interactions in both physical (Yin, Shaw, and Yu 2011) and virtual (Yu and Shaw 2008) spaces, mapping people's activity patterns (Kwan 1999), choosing destinations (Scott and He 2012), and modeling transport systems (Winter and Raubal 2006), as well as measuring accessibility.

\subsection{Accessibility}

Accessibility-related research has thrived during the last few decades, with new data and computational tools facilitating innovations rooted in classical approaches (Levine et al. 2012; Neutens, Schwanen, and Witlox 2011; Li et al. 2011; Harris 2001; Miller 1999; Burns 1979; Weibull 1976; Ingram 1971; Hansen 1959; Shen 1998)While precise definitions vary across disciplines and analytical contexts, generally accessibility is thought to mean the ease with which activities can be reached in space. Accessibility has a transportation component whereby, all else being equal, more diverse, faster, and more efficient transportation systems improve accessibility. It can take on positive or normative flavors depending on how measures are implemented (Páez, Scott, and Morency 2012). And quite often, accessibility is parsed based on the perspective of how it is measured (Kwan 1998). Place-based metrics capture the accessibility assumed to be available at a given location ( $\mathrm{Li}$ et al. 2011; Horner 2004). For example, these metrics can be used to gauge how many employment opportunities may be visited by public transit from a specific location within in a city (Horner and Mefford 2005). Gravity or potential models are among the most widely recognized place-based measures, as they incorporate distance decay as a means of discounting opportunities that are farther away from the accessibility reference points (Pooler 1987). Place-based measures can be used to assess the accessibility impacts of new or major changes to transportation infrastructure (Linneker and Spence 1992; Xie and Levinson 2011), for example. They have also found wide use in social policy contexts (Shen and Sanchez 2005), as they are readily quantified and mapped to locations such as network nodes and/or census geographies.

However, these place-based measures do not account for personal differences that would preclude an individual from experiencing their suggested measure of accessibility (Miller 2007). By definition, place-based measures assume that all people at a particular location have the same level of accessibility, when in fact personal constraints may limit one's ability to participate in activities. For instance, it 
could be the case that an individual does not have the time available to reach some of the opportunities assumed accessible by place-based measures. In this way, people-based measures of accessibility allow one to account for individual constraints on movement, activity participation, and other contextual factors that shape transportation outcomes (Neutens et al. 2010; Kwan and Weber 2003; Kim and Kwan 2003). As suggested above, many recent people-based accessibility approaches build on the timegeographic framework of Hägerstrand (1970) and incorporate such conditions within the enabling confines of GIS (Miller 1991; Kim and Kwan 2003; Neutens, Schwanen, and Witlox 2011; Yu and Shaw 2008). More robust visualization capabilities (Yu and Shaw 2008) coupled with the availability of high-resolution spatial movement data (Chen et al. 2011) has fueled these developments. However, one limitation of assessments using people-based approaches involves their idiosyncratic nature, insofar as generalization across individuals (aggregation) and related questions of (re)mapping individual metrics to places remains elusive, with only modest progress to date (Delafontaine, Neutens, and Van de Weghe 2012; Neutens, Schwanen, and Witlox 2011; Kwan 1998; Miller and Wu 2000).

\subsection{Related work and concepts}

Several efforts blending time geographic concepts and the measurement of accessibility set a context for our current aims, since our research emphasizes modeling with individual mobile objects. Building on Lenntorp (1976) and Burns (1979), Miller's (1991) work using GIS was the first to operationalize Hägerstrand's space-time prisms for the measurement of accessibility (Burns 1979; Lenntorp 1976; Miller 1991). Later research by Miller $(1999,2000)$ established theory and computational procedures for implementing space-time accessibility measures in a GIS framework with real-world-sized problem data (Miller and Wu 2000; Miller 1999). Kwan (1998) compares person- and place-based accessibility measures' suitability for measuring the accessibility of sample travelers in Columbus, Ohio, and found wide divergence in what the metrics captured. Neutens et al. (2010) extend Kwan (1998) and Miller's (1999) research in an analytical comparison of the largest set of accessibility metrics to date. In these and related papers, activities occur at a set of point locations on the landscape, with some activities being fixed or mandatory (e.g., being at work) and other activities being discretionary (e.g., shopping) in that they could ostensibly be carried out at more than one location. Thus, the thrust of much of the literature is to focus on measuring people's accessibility to discretionary activities given fixed mandatory points anchoring their potential trips (Neutens, Versichele, and Schwanen 2010; Kim and Kwan 2003; Delafontaine, Neutens, and Van de Weghe 2012; Miller and Wu 2000). Another useful observation is that accessibility measurements can be performed retrospectively for people's travel that has already occurred, such as when it is recorded in a survey (e.g, Kwan 1998), or it can be assessed prospectively for travel that has not yet happened, such as in the context of personal trip planning (Neutens, Versichele, and Schwanen 2010). Regardless of the exact accessibility application in question, most person-based metrics depend to some extent on the potential path area or tree. If the potential path areas (or trees) are combined for an individual's travel over the course of the day, these are known as daily potential path areas (trees) (Kim and Kwan 2003; Neutens, Schwanen, and Witlox 2011).

Downs and Horner (2012) altered the classical potential path tree to delineate areas within it that were more likely to be traversed by a mobile object. Their "probabilistic" potential path trees are such that locations closest to the shortest path between control points are deemed most likely to have been traversed, with those locations on the shortest path receiving the highest likelihood quantification. Similar to classical potential path trees, probabilistic potential path trees can be aggregated to produce a composite or "daily" probabilistic tree for an object that has made multiple stops. They are also applied retrospectively to analyze travel that had already occurred, as opposed to a prospective (choice-based) scenario like that which is outlined above. Their analysis also treated all control points as places of fixed 
activities or mandatory stops (they came from recorded GPS coordinates) in order to measure where the vehicle was most likely located while moving in the interim. In this way, discretionary stops (or activities) were not differentiated. The next section extends the work of Downs and Horner (2012) to formulate a new accessibility metric that is based on individual movements, is mapped to the place, and may be aggregated over individuals or locations.

\section{$3 \quad$ Model development}

Recent work by Downs and Horner (2012) developed network-based TGDE for use with mobile objects in network space. It is used to produce a measure of probability density that indicates where on a network a mobile object was most likely to be, and is computed as:

$$
\begin{aligned}
& \hat{f}_{t}(x)=(N-1)^{-1} \sum_{i=1}^{N-1} P P T^{*}\left(\frac{t_{p}(i, x)+t_{p}(x, j)}{t(i, j)-t_{a}(i, j)}\right) s_{i j}^{-1} \\
& f_{t}(x)=\text { time-geographic estimate at location } x \text { on a network, } \\
& N=\text { number of control points in dataset; consecutive points denoted } i \text { and } j, \\
& P P T^{*}=\text { distance weighting function of the potential path tree, } \\
& t(i, j)=\text { time elapsed between control points } i \text { and } j, \\
& t_{a}(i, j)=\text { time spent for a stationary activity between control points } i \text { and } j, \\
& t_{p}(,)=\text { minimum travel time between two network locations, as estimated from maximum } \\
& \quad \text { velocities along shortest path } p \text {, and } \\
& s_{i j}=\text { dimension of potential path tree for control points } i \text { and } j .
\end{aligned}
$$

Per Downs and Horner (2012), the metric in equation 1 produces a probability density, or intensity, which indicates the relative likelihood that an object was at a particular location on the network. Note that this does not express the raw probability of finding the object at a location; rather it provides a relative measure of that likelihood, which that can be directly compared across all locations in the map (Silverman 1986). Equation 1 assumes that the intensity at a given location $x$ is a function of evaluating a series of control points ( $i-j$ pairs) for a mobile object. Locations $x$ are places on a network where intensity is to be estimated; these could be network links, evenly spaced segments along links, or network nodes. $P P T^{*}$ denotes a bounded distance-weighting function that is used to (a) ensure that only locations $x$ that occur on the potential path tree will receive positive intensity values and (b) govern the relative amount of intensity assigned to locations $x$ that are on the potential path tree but not on the shortest path between $i$ and $j$ (Horner, Zook, and Downs 2012). As described in Horner et al. (2012), an exponential, linear, or other decay function can be used to model the level of intensity assigned to non-optimal paths, whereby increasingly suboptimal paths between $i-j$ receive some diminishing level of intensity based on the functional parameterization of $P P T^{*}$.

Terms in the large parentheses track the ratio of time it takes to reach a given location $x$ from the two control points to the total travel budget available for these two control points. $s_{i j}$ denotes a correction factor applied to adjust the intensity estimates downward for each $i-j$ pair controlling for the pairs' different spatial structures, equivalent to the number of unique shortest connection paths (Downs and Horner 2012). The scalar in front of the summation sign averages the intensity at any location $x$ by dividing by the total number of potential path trees. For a fuller accounting on the basic motivations and aims of TGDE, readers are referred to Downs (2010), Downs and Horner (2012), and Horner et al. 
(2012) for additional discussion of possible parameterizations of $P P T^{*}$ and the factor $s_{i j}$.

In reconstructing travelers' "missing destinations," Horner et al. (2012) reduce equation 1 to focus on a single $i-j$ point pair (probabilistic potential path tree) for one individual:

$$
\hat{f}_{t}(x)^{\prime}=P P T^{*}\left(\frac{t_{p}(i, x)+t_{p}(x, j)}{t(i, j)-t_{a}(i, j)}\right)
$$

In equation 2, summations and the dimensionality adjustment $s_{i j}$ are omitted. The result of equation 2 mapped for all locations $x$ in the $i-j$ pair's potential path tree reveals locations the object most likely traversed.

We adapt equation 2 to visualize an object's accessibility levels along the tree relative to specific opportunities or activities. We combine estimates on the likelihood of the object being at a place, with the magnitude of activities at that place. First, we alter equation 2 to denote the time geographic estimate for a given object for a given object $q$ at the $k^{t h}$ location $x$ :

$$
\hat{f}_{t}\left(x_{q k}\right)^{\prime}=P P T^{*}\left(\frac{t_{p}\left(i, x_{q k}\right)+t_{p}\left(x_{q k}, j\right)}{t(i, j)-t_{a}(i, j)}\right)
$$

With this intensity estimator (probabilistic potential path tree) defined for a single $i$-j pair, we must also consider a term capturing the attractiveness of the activities at location $k$, or $O_{k}$. Let $O_{k}$ be bounded such that $0 \leq O_{k} \leq 1$ and $\sum_{k} O_{k}=1$, implying that $O_{k}$ represents the $k^{t h}$ location's fraction of the region's total activties. Thus, we define the object's accessibility level to an activity along the tree at location $x$ as: $\hat{f}_{t}\left(x_{q k}\right)^{\prime} O_{k}$

In equation 4, higher accessibility values result from larger intensity scores at locations that have larger shares of regional activities. For an object making multiple stops, we compute a composite accessibility value at each $x$ location by summing accessibility scores for each $i-j$ pair.

$$
A_{q k}=\sum_{i=i}^{N-1} \hat{f}_{t}\left(x_{q k}\right)^{\prime} O_{k}
$$

Now we reintroduce the correction factor $s_{i j}$ to account for the different dimensionalities of $i-j$ pairs (see Downs and Horner 2012), as well as the scalar for density estimation,

$$
A_{q k}=(N-1)^{-1} \sum_{i=i}^{N-1} \hat{f}_{t}\left(x_{q k}\right)^{\prime} s_{i j}^{-1} O_{k}
$$

$A_{q k}$ defines the composite accessibility score for object $q$ at location $k$. It is bounded from 0 to 1 , with higher values indicating greater accessibility. From this, we define the total accessibility score for mobile object $q$ as

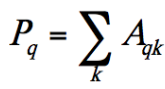

and when equation $5 \mathrm{~b}$ is estimated for multiple moving objects, then it is possible to compute the 
total accessibility of all objects to an activity at the kth location as

$$
S_{k}=\sum_{q} A_{q k}
$$

An example accessibility analysis with the TGDE metric is demonstrated using sample travel diary data items for hypothetical vehicular trips. This particular traveler makes three stops after leaving his or her home location and then returning. Figure 1 shows the vehicle stops and TGDE intensity values based on a linear weighting function input for $P P T^{*}$ (equation 1). Darker areas correspond to locations where the vehicle was more likely to have traveled given its time budget. The red route structure linking stops in the figures represents the shortest path between known stops but not does not necessarily indicate the path that was taken. Figure 2 displays synthetic opportunity data. The traveler's composite potential path tree consisted of 3145 network nodes. Three hundred of them were randomly selected, and then randomly assigned each a high, medium, or low level of attractiveness (scored 3, 2, and 1, respectively). These were converted to node-based opportunity levels such that . $\sum_{k} O_{k}=1$ Figure 3 shows accessibility values for the traveler (scaled by $10^{5}$ to reduce excess decimals). Larger, darker nodes are places where the traveler was more likely to be and encounter higher-attractiveness activities. The traveler's total accessibility score is 0.7644 (equation 6).

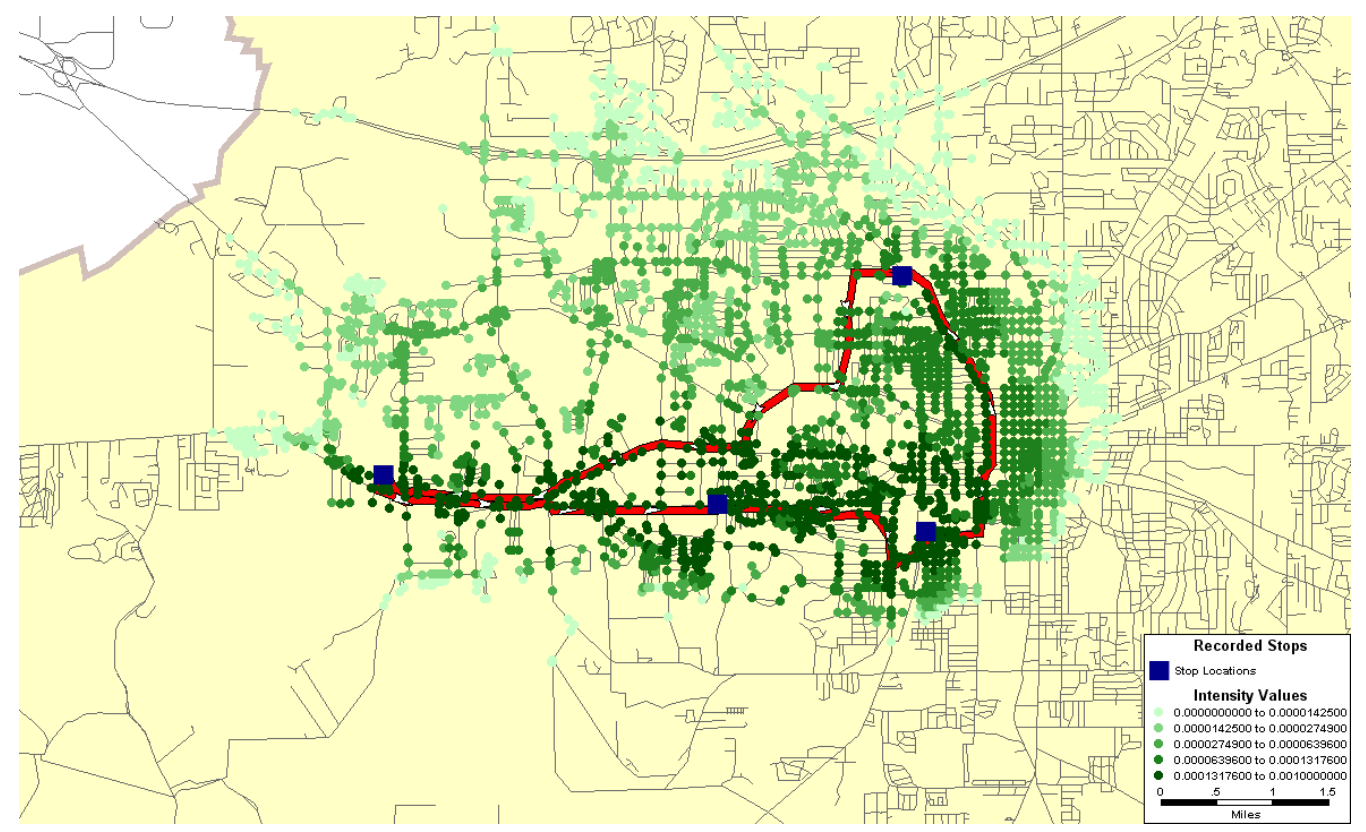

Figure 1: Intensity values for Traveler 1 (equation 1). 


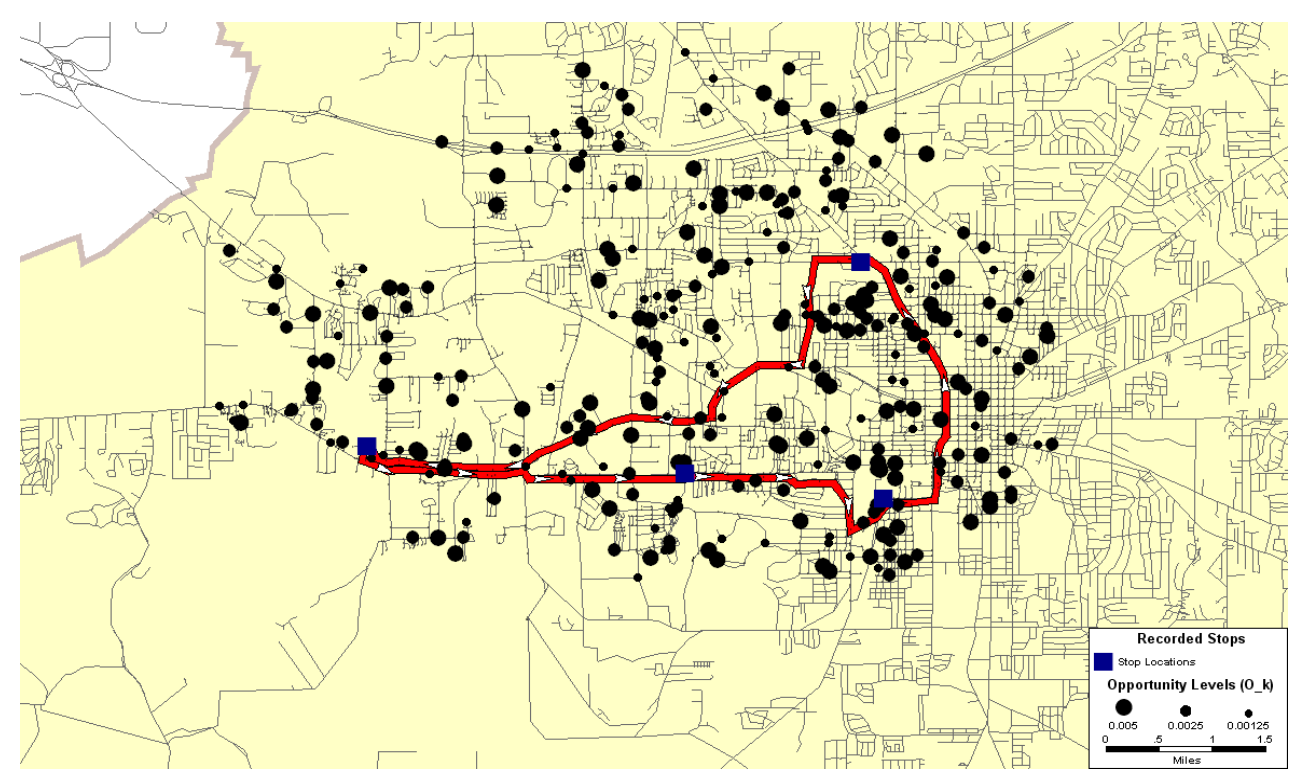

Figure 2: Activities distribution $\left(O_{k}\right)$.

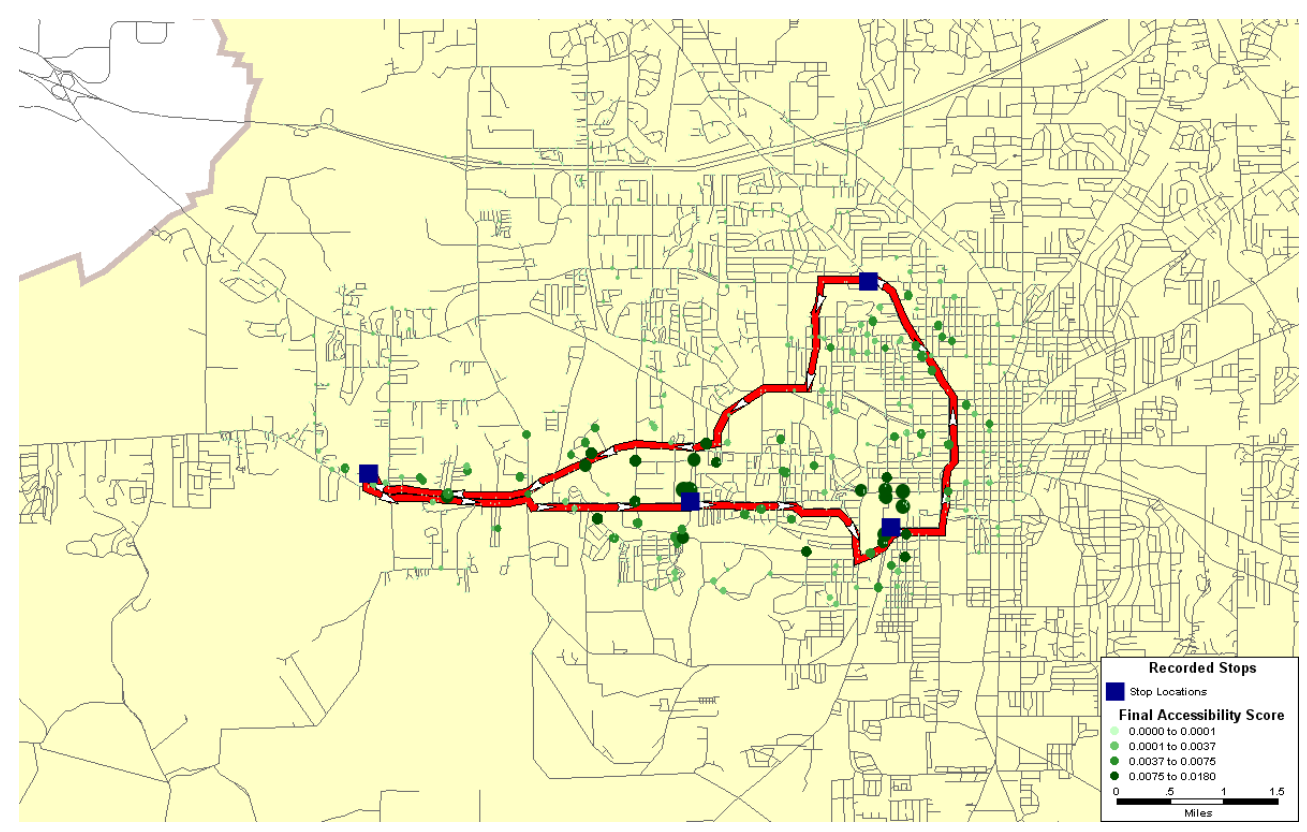

Figure 3: Accessibility for traveler $\left(A_{q k}\right)$.

Looking at the figures in concert with their respective equations, $A_{q k}$ should be viewed as the average accessibility experienced by object $q$ at location $k$. This is because an object may have a given location $k$ in multiple of its potential path trees (equation 2), but their cumulative accessibility is averaged at $k$ per the reintroduction of the scalar $(N-1)^{-1}$ in equation 5b. Therefore, equation 6 returns the cumulative average accessibility of the individual, while equation 7 represents the cumulative average accessibility at a given location.

To place our model in context, it is useful to consider the work of Weibull (1976) and some subsequent elaborations by Burns (1979), Miller (1999), and Neutens et al. (2010). Weibull (1976) set out basic requirements for attraction-based accessibility measures, which have been adapted to guide development of individual space-time accessibility measures under personal budget constraints (Burns 1979, 
Miller 1999). Although we offer no formal mathematical proof here, our accessibility measure is consistent with the tenets of that framework. Moving to a more granular level, it is instructive to examine the comprehensive accessibility tests described recently in Neutens et al. (2010). There, they present several models, including an extension of Lenntorp's (1976) work, which accounts for the number of activities within a person's potential path tree (named NUMD). This approach uses a decay function to differentially weight locations in the tree based on the time it takes to reach them, given the locations of the anchor points. They describe a second model of interest that is rooted in the framework of Miller (1999) and Burns (1979), which incorporates a measure of activities' attractiveness (named BAGG). It is used to sum the aggregate accessibility benefit to individuals since it accounts for the total choice opportunities available within the daily potential path area (tree). Like the previously discussed NUMD model, a distance decay parameterization is included to differentially weight locations that are farther away from anchor points. Comparing our approach to these, like NUMD and BAGG, we are able to differentially weight areas within potential path trees using decay functions (per PPT*). However, we do this weighting given the ratio of the travel time it takes to visit a point to the total time budget available to the traveler. Both BAGG and NUMD differentially weight locations based on the average time it takes to visit them relative to the two anchor points (Neutens et al. 2010). Another key difference is the application of the metrics, whereby NUMD and BAGG could be applied retrospectively to analyze travel that has happened or prospectively to analyze potential travel choices. Both metrics differentiate between mandatory and discretionary activities and measure accessibility based on the possible participation in the latter. In our approach, analysis is carried out retrospectively and in keeping with the sensibilities of mobile object analysis, all known stops are considered fixed or mandatory stops and accessibility to opportunities is assessed between all points in an object's travel record. Effectively, there are no discretionary activities the object (e.g., traveler) is assuming to choose from, but rather the model assesses the accessibility to other opportunities en route between any revealed choices. Lastly, a final and quite substantial difference between measures such as NUMD and BAGG and our approach is that both NUMD and BAGG are resolved to the individual. That is, they produce an accessibility score for the individual. Our model, however, begins by producing an individually based metric for locations (equation $5 \mathrm{~b}$ ) that in turn may be aggregated to the individual level (equation 6) or aggregated over multiple individuals that traverse the same location (equation 7). These features of our model are explored in the next section.

\section{$4 \quad$ Data and analytical details}

We seek to demonstrate the use of our new metric in a realistic activity analysis scenario where we assess people's accessibility to opportunities given constraints on their movement. These constraints follow from mandatory activities people undertook, their time available, and the characteristics of the transportation facilities supporting travel. The goal is to visualize and quantify the nature of the opportunities that people are able to access, which in this case will be employment opportunities relative to their daily travel commitments.

\subsection{Study area and network data}

For our study area, we chose to work in Leon County, Florida. Leon County contains Florida's capital city of Tallahassee and is home to three major institutions of higher learning, in addition to a number of state government installations and private employers. According to the 2010 U.S. Census, the population of Leon County was approximately 275,487.

Road network data for Leon County were compiled from Census TIGER line files, which contain the range of facilities from interstates to local neighborhood streets. Census functional classification 
codes appearing in the database were used to infer speed limits for each roadway linkage, which in turn were used to assign free-flow travel times. Then, each linkage was subjected to an additional time increase of approximately 30 percent to account for congestion and other possible delay effects.

\subsection{Travel activity data}

Synthetic activity data were used in our analyses. There has been no travel survey conducted in the Tallahassee area during the last several years that would facilitate our research. Our synthetic data were designed to follow a typical travel diary-type format with activity locations recorded for 11 individuals making a total of 40 trips. Eight of the eleven individuals were a part of multi-person households and shared origin locations. The number and location of each individual's trip destinations were selected at random from within the study area. All tours were assumed to begin and end at the individual's home location, and, as such, the first network node selected for any individual was assumed to be the home. Times between activity stops were estimated as the shortest paths between those locations based on the road network identified above. In turn, a given individual's activity (time) budget for a given trip segment was assumed to be 1.5 times that of the shortest path between the origin-destination pair, which is not unreasonable given recent empirical evidence (Papinski and Scott 2013). For example, if the shortest path between two stops in an individual's itinerary took 3 minutes to traverse, for purposes of implementing our accessibility model, we would assume this individual had a total time budget of 4.5 minutes with which to work. This allows for the possibility that the individual's trip could have deviated from the exact shortest path. It also means that some locations off the individual's shortest path will be assigned some positive intensity (and possibly accessibility). Additional details about the construction and characteristics of these synthetic travel data can be found in Horner et al. (2012). Ideally we would have empirical activity data with which to work, as these would be the best representation of actual human mobility patterns. However, we feel these synthetic data are sufficiently representative of realistic activity patterns to be useful for illustrating the metrics.

\subsection{Opportunity data}

We utilized employment information accessed from the Census Longitudinal Employment Household Dynamics (LEHD) database. LEHD represents a partnership between the Census and other government agencies to provide timely information on workforce characteristics (Horner and Schleith 2012). Through the OnTheMap application (http://onthemap.ces.census.gov/), spatial information on employment characteristics for multiple geographical scales in the U.S. is accessible. We used OnTheMap in April 2012 to extract job locations within Leon County for the year 2010. These data consist of 162,355 primary and secondary jobs resolved to the census block scale (Leon County contains 6198 census blocks). For purposes of the analysis, job counts are associated with the centroid of their respective census block, and, in turn, these centroid points are associated in GIS with the road network. Job counts in each block are divided by the total number of jobs in the study region to represent proportions, consistent with the definition of $O_{k}$ described above. The jobs data are mapped in Figure 4 along with the travel activity data. 


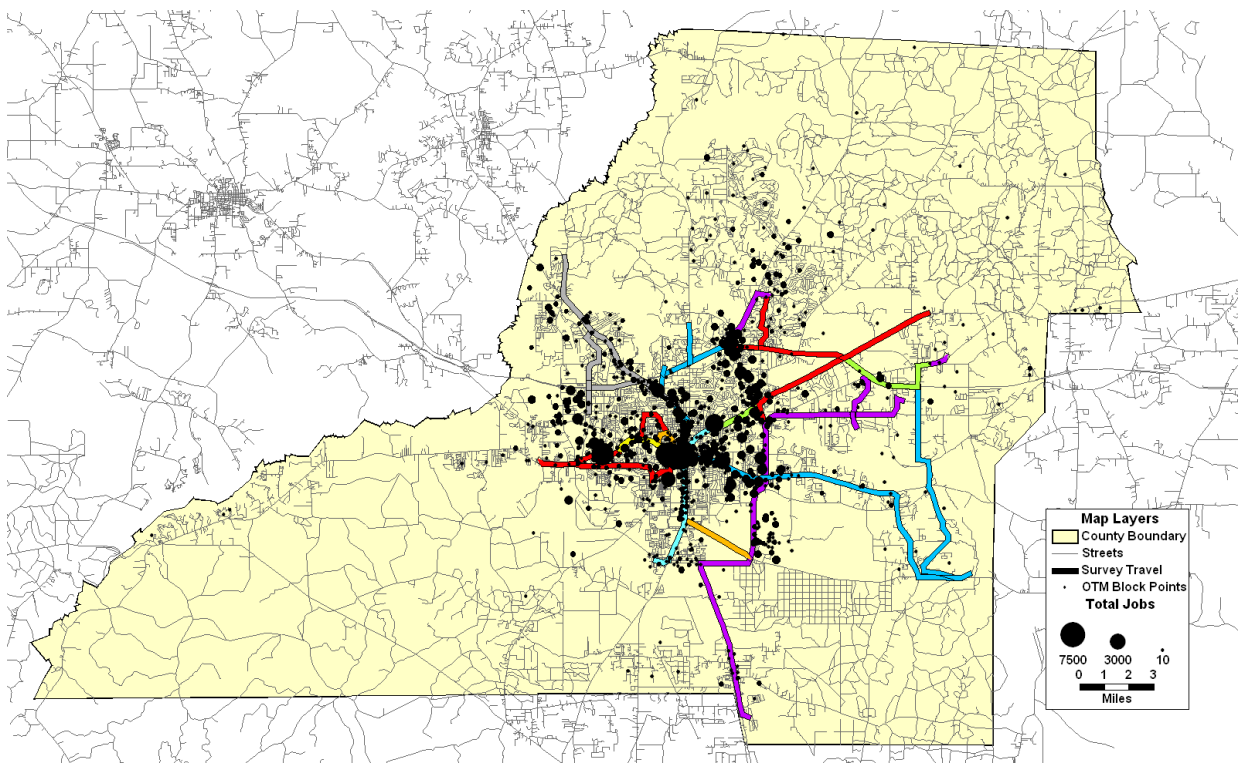

Figure 4: Leon County study area with travel and jobs data.

Many efforts over the years have analyzed accessibility to jobs (Sanchez, Shen, and Peng 2004; Shen 1998; Wang 2003). If one views our analysis through the lens of people's potential job searches, then clearly these job locations represent employment opportunities that are "taken." From that standpoint, existing jobs should be thought of as proxies for the location of additional employment opportunities. Moreover, employment locations also are proxies for other types of activity locations, since food opportunities, service, and retail establishments tend to cluster in cities and often along major thoroughfares.

\subsection{Computational environment}

A combination of software packages was used to implement the TGDE accessibility measures. Spatial data management was undertaken with ArcGIS version 10 and TransCAD version 4.7. Python scripting and Microsoft Excel were utilized to operationalize the network based TGDE accessibility metric described in equation 5b. All estimates of accessibility and intensity follow from the node-based framework described in Horner et al. (2012). Other representational strategies are possible to estimate TGDE-derived metrics, including the possibility using of the line pixel or lixel (Xia and Yan 2008). But here- given our interest in including opportunities that occur at nodes, as well as the computational advantages inherent to working with nodes—we proceed using nodes as our estimation basis.

\section{$5 \quad$ Results}

Summary results from our analysis of the 11 travelers' activity data appears in Table 1. Travelers are identified (Traveler ID) by their "household" number first, and their "person" number second, separated by an underscore. Accessibility surfaces were created for each traveler based on equation $5 \mathrm{~b}$ using a linear decay function such that "1-" is substituted for $P P T^{*}$. These values were summed for each traveler, per equation 6, and each person's accessibility scores are reported in the table. For comparison purposes, we also computed the percentages of study area jobs within the cumulative (daily) traditional potential path trees of each traveler. 
Table 1: Summary of accessibility results.

\begin{tabular}{lrrrr} 
Traveler ID & Pct. of Jobs in PPT & Ranking & Acc. Score $\left(\boldsymbol{P}_{q}\right)$ & Ranking \\
\hline 1_1 & $46.29 \%$ & 7 & 0.382 & 5 \\
2_1 & $96.23 \%$ & 1 & 0.103 & 11 \\
3_1 & $56.13 \%$ & 5 & 0.483 & 4 \\
3_2 & $60.23 \%$ & 3 & 0.333 & 6 \\
4_1 & $95.18 \%$ & 2 & 0.243 & 9 \\
4_2 & $39.70 \%$ & 8 & 0.691 & 1 \\
$5 \_1$ & $9.93 \%$ & 10 & 0.622 & 2 \\
6_1 & $58.87 \%$ & 4 & 0.570 & 3 \\
6_2 & $51.04 \%$ & 6 & 0.260 & 7 \\
7_1 & $25.59 \%$ & 9 & 0.130 & 10 \\
7_2 & $3.35 \%$ & 11 & 0.248 & 8
\end{tabular}

The results of each traveler's potential path tree assessment demonstrates a wide range of values whereby the smallest percentage of jobs accessible to a traveler given their time budget was 3.35 percent (traveler 7_2), while the largest percentage of jobs accessible to a traveler was 96.23 percent (traveler 2_1). Again, this is a traditional potential path tree calculation where a given opportunity (job) location was deemed accessible if it was reachable within the individual's time budget. To facilitate comparison, individual travelers are assigned rankings based on this percentage whereby higher percentages of accessible jobs result in higher rankings. Considering each traveler's cumulative density-based accessibility score $\left(P_{q}\right)$, a different assessment of accessibility emerges. The travelers that scored higher on the traditional potential path tree measure did not necessarily have a high cumulative density-based score. Individual's accessibility scores $\left(P_{q}\right)$ were also ranked in descending order, revealing several prominent changes in rankings (e.g., traveler 2_1 was ranked first based on the potential path tree metric but eleventh on the density-based measure). Other travelers experience similar shifts in rank when comparing the two measures.

The reasons for these shifts are twofold, and follow from our prior discussion of the models. First, because the accessibility approach accounts for the differing amounts of time it takes to reach various locations in the potential path tree, not all locations within the tree will be given equal weight. Generally speaking, those locations farther from the shortest path connecting a given $i-j$ pair will receive less intensity (and accessibility). Secondly, because the accessibility value at a given location $x$ (equation 5a) is subsequently scaled per equation $5 \mathrm{~b}$, the more complex the traveler's itinerary (as $N$ grows), the more accessibility at that location will diminish, all else being equal. In practical terms, what this means is that a traveler could pass by (and easily reach given their time budget) large employment clusters early in their itinerary, but later traverse locations that were not proximal to employment clusters. The "averaging" that then happens as a function of equation $5 \mathrm{~b}$ would serve to reduce the accessibility scores at those early locations $x$, which in turn would decrease the individual's overall personal accessibility score $\left(P_{q}\right)$. Clearly then, $P_{q}$ represents a much more conservative assessment of accessibility than its counterpart based on the classical potential path tree. This echoes findings elsewhere suggesting the benefits of developing measures able to articulate the heterogeneous nature of accessibility among individuals (Neutens et al. 2010)

One of the other key features of our density-based approach is the ability to summarize the accessibility experienced by a cumulative group of travelers at a given location $x$. This is expressed in equation 7 as $S_{k}$ and is mapped in Figure 5 based on the individual itineraries of all 11 travelers. 


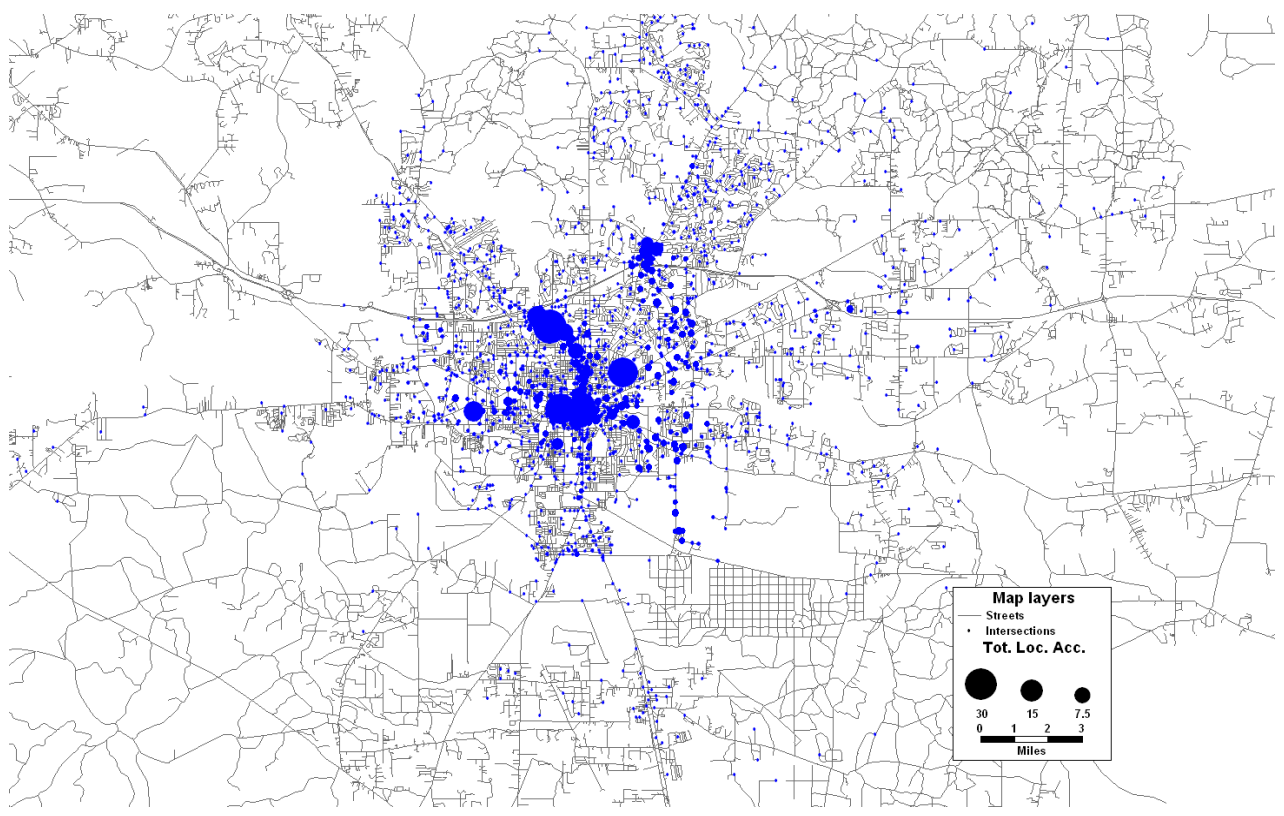

Figure 5: Cumulative aggregate job accessibility $\left(S_{k}\right)$.

The figure shows the locations in the study area that had the highest cumulative accessibility scores as they were aggregated from individual's experiences. Due to their relatively large number of opportunities combined with their proximity to the travelers' fixed stops, a clear north-south corridor of high accessibility scores emerges on the map. To the north, this appears to be bounded by the interstate highway (I-10). To the south, this high-accessibility regime stretches into the downtown area. This area is known as the Monroe Street corridor and is a major thoroughfare with a number of restaurants, shopping areas, light commercial, and other employment-rich land uses. Comparing this map to the prior figure depicting purely employment opportunities (Figure 4), it is apparent that some of the more prominent job locations on both the east and west side of Tallahassee diminish in relative importance when we look at how accessible they were to this group of travelers.

\section{Discussion and conclusions}

Rooted in contemporary work in mobile objects tracking, density estimation, and time-geography, this paper has sought to develop a new approach to conceptualizing and measuring accessibility. Reflective of its "mobile-objects" origins, the measure is used to retrospectively analyze an object's travel itinerary (e.g., a vehicle) and quantify levels of accessibility experienced along its route. As we have pointed out, there are some similarities between our measure and existing approaches (Neutens et al. 2010) However, the context surrounding how our model is applied (i.e., retroactively assuming all recorded activity stops are mandatory) and the nature of how it quantifies and maps accessibility values to locations sets it apart from current models. In this way, it should not be thought of as necessarily better (or worse) than existing approaches, but rather as a way of capturing accessibility from a different (i.e., mobile objects) perspective. Our model lends itself to the analysis of other transportation modes such as bicycling or pedestrian trips, since people using these modes are also mobile objects.

One of the more interesting aspects of our work is its implications for the people-based vs. placebased accessibility divide present in the literature. As detailed earlier in the paper, both measurement approaches have their strengths and weaknesses and are geared toward different analytical contexts (Burns 1979; Ingram 1971; Miller 1999; Pooler 1987; Li et al. 2011), and there has been limited success at 
uniting the two (Miller and Wu 2000; Kwan 1998; Delafontaine, Neutens, and Van de Weghe 2012). At some level, our work would seem to bridge these perspectives, as the basic location-based measure of equation $5 \mathrm{~b}$ is derived from the accessibility score of an individual. Moreover, one can aggregate the scores of individuals traversing the same locations to derive aggregate location-based accessibility scores. In theory, these capabilities allow one to accomplish tasks common to both the individual- and place-based domains. From the individual perspective, we have accounted for personal constraints by using the full record of the traveler, and from the place-based perspective, we can map accessibility to the location.

There are many potential policy applications of our approach. For example, Miller and Wu (2000) suggest that a robust and composite accessibility measure should be able to determine how a hypothetical change to a roadway facility could affect accessibility. Certainly this and other types of policy questions can be tackled using our metric, as changes in the underlying transportation system would affect individuals' route choice options and in turn impact their accessibility. For instance, alterations to a road network, such as adding lane capacity, would change travel times and affect the ease of movement. The before and after accessibility levels for individuals could then be quantified and aggregated if desired. Similar logic could be applied in policy applications where one wishes to assess the effects of major changes to employment or other activity locations on accessibility.

The scope of possible future work with our metric is wide open. One area for experimentation is to examine multiple visualization strategies for displaying location-based accessibility outputs. Our nodebased approach is relatively computationally efficient and effectively conveys difference, but a link or sub-link (lixel) approach is perhaps a more elegant way of mapping the metric (see Downs and Horner 2012). We would also like to use our metric with a larger data set consisting of more travelers making multimodal trips. Here, our research assumed all travel was by vehicle, which is not unrealistic given that the auto share of trips in Tallahassee is more than 90 percent. Applying our model in a multimodal context would offer additional insights into how transportation systems facilitate accessibility.

It is also worth reiterating that the metrics were demonstrated with synthetic data, which presents certain limitations in terms of the study's findings. Here, the results suggest that the new metrics capture accessibility in a different way than the traditional potential path approach used in the comparisons. And while intuitively there are logical reasons why this should be the case, some of these contrasts may not hold if the indices are further tested on other datasets consisting of individuals with different travel patterns. Thus, future work should look to further explorations of these accessibility models with larger, empirically derived datasets. Related to this, a larger data set would also likely have information about individual travelers' socioeconomic and personal characteristics. In that case, after accounting for day-to-day travel variability (Kang and Scott 2010), it would be interesting to look at the relationships between the accessibility levels of individuals based on our metric and their personal characteristics. This would have implications in areas such as health, where there would be interest in knowing how people use space and are able to access opportunities (e.g., healthy food) given their personal constraints and socioeconomic characteristics (Widener et al. 2013). Lastly, many studies control for the opening hours of facilities in determining accessibility (Neutens, Schwanen, and Witlox 2011), which is something we did not do here. This could be attempted in future studies and would serve to add more conservatism to estimating accessibility with our density-based measure. In summary, these and other opportunities are worth pursuing in our efforts to conceptualize and measure accessibility. 


\section{$7 \quad$ Acknowledgments}

Portions of this research were supported by grants made by the National Science Foundation (NSF) to Horner (BCS-1062924) and Downs (BCS-1062947). Any opinions, findings, and conclusions or recommendations expressed here are those of the authors and do not necessarily reflect the views of NSF. The authors would like to thank Mr. Brandon Zook (Florida State University) for his research assistance with implementing a version of the network-based TGDE metric in equation 1 and computing TGDE surfaces for records in the Tallahassee synthetic data.

\section{References}

Burns, L. D. 1979. Transportation, Temporal, and Spatial Components of Accessibility. Lexington, MA: Lexington Books.

Chen, J., S. L. Shaw, H. B. Yu, F. Lu, Y. W. Chai, and Q. L. Jia. 2011. Exploratory data analysis of activity diary data: a space-time GIS approach. Journal of Transport Geography 19(3): 394-404. doi:10.1016/j.jtrangeo.2010.11.002.

Delafontaine, M., T. Neutens, and N. Van de Weghe. 2012. A GIS toolkit for measuring and mapping space-time accessibility from a place-based perspective. International Journal of Geographical Information Science 26(6): 1131-1154. doi:10.1080/13658816.2011.635593.

Downs, J. A. 2010. Time-geographic density estimation for moving point objects. Lecture Notes in Computer Science 6292: 16-26. doi:10.1007/978-3-642-15300-6_2.

Downs, J. A., and M. W. Horner. 2012. Probabilistic potential path trees for visualizing and analyzing vehicle tracking data. Journal of Transport Geography 23: 72-80. doi:10.1016/j.jtrangeo.2012.03.017.

El-Geneidy, A., and D. Levinson. 2007. Mapping accessibility over time. Journal of Maps 3(1): 76. doi:10.1080/jom.2007.9710829.

Hägerstrand, T. 1970. What about people in Regional Science? Papers of the Regional Science Association 24(1): 6-21. doi:10.1007/BF01936872.

Handy, S. L., and D. A. Niemeier. 1997. Measuring accessibility: An exploration of issues and alternatives. Environment and Planning A 29(7): 1175-1194. doi:10.1068/a291175.

Hansen, W. G. 1959. How accessibility shapes land use. Journal of the American Institute of Planners 25(2): 73-76. doi:10.1080/01944365908978307.

Harris, B. 2001. Accessibility: Concepts and applications. Journal of Transportation and Statistics 4(3): $15-30$.

Horner, M. W. 2004. Exploring metropolitan accessibility and urban structure. Urban Geography 25(3): 264-284. doi:10.2747/0272-3638.25.3.264.

Horner, M. W., and J. N. Mefford. 2005. Examining the spatial and social variation in employment accessibility: A case study of bus transit in Austin, Texas. In Access to Destinations, edited by D. Levinson and K. Krizek, 193-214. Oxford: Elsevier.

Horner, M. W., B. Zook, and J. A. Downs. 2012. Where were you? Development of a time-geographic approach for activity destination re-construction. Computers, Environment and Urban Systems 36(6): 488-499. doi:10.1016/j.compenvurbsys.2012.06.002.

Horner, M. W., and D. Schleith. 2012. Analyzing temporal changes in land use-transportation relationships: A LEHD-based approach. Applied Geography 35(1-2): 491-498.

Hornsby, K., and M. J. Egenhofer. 2002. Modeling moving objects over multiple granularities. Annals of Mathematics and Artificial Intelligence 36(1): 177-194. doi:10.1023/A:1015812206586.

Ingram, D. R. 1971. The concept of accessibility: A search for an operational form. Regional Studies 5(2): 101-107. doi:10.1080/09595237100185131. 
Kang, H., and D. M. Scott. 2010. Exploring day-to-day variability in time use for household members. Transportation Research Part A: Policy and Practice 44(8): 609-619. doi: 10.1016/j.tra.2010.04.002.

Kim, H., and M. P. Kwan. 2003. Space-time accessibility measures: A geocomputational algorithm with a focus on the feasible opportunity set and possible activity duration. Journal of Geographical Systems 5(1): 71-91. doi:10.1007/s101090300104.

Kuijpers, B., H. J. Miller, T. Neutens, and W. Othman. 2010. Anchor uncertainty and space-time prisms on road networks. International Journal of Geographical Information Science 24(8): 1223 1248. doi:10.1080/13658810903321339.

Kwan, M. P. 1998. Space-time and integral measures of individual accessibility: A comparative analysis using a point-based framework. Geographical Analysis 30(3): 191-216. doi:10.1111/j.1538-4632.1998. tb00396.x.

. 1999. Gender and individual access to urban opportunities: A study using space-time measures. Professional Geographer 51(2): 210-227. doi:10.1111/0033-0124.00158.

Kwan, M. P., and J. Weber. 2003. Individual accessibility revisited: Implications for geographical analysis in the twenty-first century. Geographical Analysis 35(4): 341-353. doi:10.1111/j.1538-4632.2003. tb01119.x.

Laube, P., S. Imfeld, and R. Weibel. 2005. Discovering relative motion patterns in groups of moving point objects. International Journal of Geographical Information Science 19(6): 639-668. doi:10.1080/13658810500105572.

Lenntorp, B. 1976. Paths in Space-time Environments: A Time-Geographic Study of Movement Possibilities of Individuals. Lund Studies in Geography, Series B, Human Geography (number 44): The Royal University of Lund, Sweden.

- 1999. Time-geography at the end of its beginning. GeoJournal 48 (3):155-158. doi:10.1023/A:1007067322523.

Levine, J., J. Grengs, Q. Y. Shen, and Q. Shen. 2012. Does accessibility require density or speed? A comparison of fast versus close in getting where you want to go in U.S. metropolitan regions. Journal of The American Planning Association 78(2): 157-172. doi:10.1080/01944363.2012.677119.

Li, Q. Q., T. Zhang, H. D. Wang, and Z. Zeng. 2011. Dynamic accessibility mapping using floating car data: a network-constrained density estimation approach. Journal Of Transport Geography 19(3): 379-393. doi:10.1016/j.jtrangeo.2010.07.003.

Linneker, B. J., and N. A. Spence. 1992. An accessibility analysis of the impact of the M25 London orbital motorway on Britain. Regional Studies 26(1): 31-47. doi:10.1080/00343409212331346761.

Miller, H. J. 1991. Modelling accessibility using space-time prism concepts within geographical information systems. International Journal of Geographical Information Science 5(3): 287-301. doi:10.1023/A:1009820006075.

1999. Measuring space-time accessibility benefits within transportation networks: Basic theory and computational procedures. Geographical Analysis 31(1): 187-212.

- 2005. A Measurement theory for time geography. Geographical Analysis 37(1): 17-45.

- 2007. Place-based versus people-based geographic information science. Geography Compass 1(3): 503-535.

Miller, H. J., and Y. Wu. 2000. GIS software for measuring space-time accessibility in transportation planning and analysis. GeoInformatica 4(2): 141-159.

Neutens, T., T. Schwanen, and F. Witlox. 2011. The prism of everyday life: Towards a new research agenda for time geography. Transport Reviews 31(1): 25-47. doi:10.1080/01441647.2010.484153

Neutens, T., T. Schwanen, F. Witlox, and P. De Maeyer. 2010. Equity of urban service delivery: a comparison of different accessibility measures. Environment and Planning A 42(7): 1613-1635. 
doi:10.1068/a4230.

Neutens, T., M. Versichele, and T. Schwanen. 2010. Arranging place and time: A GIS toolkit to assess person-based accessibility of urban opportunities. Applied Geography 30(4): 561-575. doi:10.1016/j. apgeog.2010.05.006.

O'Sullivan, D., A. Morrison, and J. Shearer. 2000. Using desktop GIS for the investigation of accessibility by public transport: an isochrone approach. International Journal of Geographical Information Science 14(1): 85-104. doi:10.1080/136588100240976.

Páez, Antonio, D. M. Scott, and C. Morency. 2012. Measuring accessibility: positive and normative implementations of various accessibility indicators. Journal of Transport Geography 25: 141-153.

Papinski, D., and D. M. Scott. 2013. Route choice efficiency: an investigation of home-to-work trips using GPS data. Environment and Planning A 45(2): 263-275.

Pooler, J. 1987. Measuring geographical accessibility - A review of current approaches and problems in the use of population potentials. Geoforum 18(3): 269-289.

Sanchez, T. W., Q. Shen, and Z. R. Peng. 2004. Transit mobility, jobs access and low-income labour participation in US metropolitan areas. Urban Studies 41(7): 1313-1331.

Scott, D. M., and M. W. Horner. 2008. Examining the role of urban form in shaping people's accessibility to opportunities: An exploratory analysis. Journal of Transportation and Land Use 1(2): 89-119. doi:10.1016/j.jtrangeo.2012.03.021.

Scott, D. M., and S. Y. He. 2012. Modeling constrained destination choice for shopping: a GIS-based, time-geographic approach. Journal of Transport Geography 23:60-71. Journal of Transport Geography 23:60-71. doi:10.1016/j.jtrangeo.2012.03.021.

Shen, Q. 1998. Location characteristics of inner-city neighborhoods and employment accessibility of low-wage workers. Environment and Planning B-Planning \& Design 25(3): 345-365.

Shen, Q., and T. W. Sanchez. 2005. Residential location, transportation, and welfare-to-work in the United States: A case study of Milwaukee. Housing Policy Debate 16(3-4): 393-431. Housing Policy Debate 16 (3-4):393-431. doi:10.1080/10511482.2005.9521551.

Silverman, B. W. 1986. Density Estimation for Statistics and Data Analysis. London: Chapman and Hall.

Wang, F. H. 2003. Job proximity and accessibility for workers of various wage groups. Urban Geography 24(3): 253-271. doi: 10.2747/0272-3638.24.3.253.

Weibull, J. W. 1976. An axiomatic approach to the measurement of accessibility. Regional Science and Urban Economics 6(4): 357-379, (4):357-379. doi: 10.1016/0166-0462(76)90031-4.

Widener, M. J., S. Farber, T. Neutens, and M. W. Horner. 2013. Using urban commuting data to calculate a spatiotemporal accessibility measure for food environment studies. Health \& Place 21: 1-9. doi:10.1016/j.healthplace.2013.01.004.

Winter, S., and Z. C. Lin. 2011. The elements of probabilistic time geography. GeoInformatica 15(3): 417-434.

Winter, S., and M. Raubal. 2006. Time geography for ad-hoc shared-ride trip planning. In Proceedings of the 7 th International Conference on Mobile Data Management. IEEE Computer Society.

Xia, Z. X., and J. Yan. 2008. Kernel density estimation of traffic accidents in a network space. Computers Environment and Urban Systems 32(5): 396-406. doi:10.1016/j.compenvurbsys.2008.05.001.

Xie, F., and D. Levinson. 2011. Evaluating the effects of the I-35W bridge collapse on road-users in the twin cities metropolitan region. Transportation Planning and Technology 34(7): 691-703. doi:10.10 80/03081060.2011.602850.

Yin, L., S. L. Shaw, and H. Yu. 2011. Potential effects of ICT on face-to-face meeting opportunities: a GIS-based time-geographic approach. Journal of Transport Geography 19(3): 422-433. doi:10.1016/j. jtrangeo.2010.09.007. 
Yu, H., and S. L. Shaw. 2008. Exploring potential human activities in physical and virtual spaces: a spatio-temporal GIS approach. International Journal of Geographical Information Science 22(4): 409-430. doi:10.1080/13658810701427569. 